\title{
Orbital Angular Momentum in QCD and its Observability through Deep Inelastic Processes
}

\author{
Simonetta Liuti ${ }^{* \dagger}$ \\ University of Virginia and Laboratori Nazionali di Frascati, Italy \\ E-mail: sl4yevirginia.edu
}

\section{Aurore Courtoy}

Departamento de Fisica, Centro de Investigacion y de Estudios Avanzados, Mexico D.F.

E-mail: acourtoy@is.cinvestav.mx

\section{Michael Engelhardt}

New Mexico State University

E-mail: engel@nmsu. edu

\section{Abha Rajan}

University of Virginia

E-mail: ar5xc@virginia.edu

\begin{abstract}
A new framework is presented for a direct measurement of the quark orbital angular momentum contribution to the proton spin. Contrary to what implied in most of the recent literature on the subject, the direct observation of quark orbital angular momentum does not require transverse spin polarization. It is instead obtained in deeply virtual Compton scattering from a longitudinally polarized proton target.
\end{abstract}

QCD Evolution 2015 -QCDEV2015-

26-30 mAY 2015

Jefferson Lab (JLAB), Newport News Virginia, USA

\footnotetext{
* Speaker.

$\dagger$ This work is in part supported by the U.S. Department of Energy, Office of Science, Office of Nuclear Physics contracts DE-AC05-06OR23177, DE-FG02-01ER4120, DE-FG02-96ER40965
} 


\section{Introduction}

After the pioneering EMC experiments [1,2] demonstrated that the proton spin is not given by the sum of the spins of the quarks, understanding the quark and gluon Orbital Angular Momentum $(\mathrm{OAM}), L_{q, g}$, in addition to the gluon spin has been a central focus of hadronic physics. Understanding OAM in the proton was the original motivation for introducing Generalized Parton Distributions (GPDs) in Refs.[3, 4], in that that they could provide a new experimental access through a class of exclusive reactions, including Deeply Virtual Compton Scattering (DVCS), Deeply Virtual Meson Production (DVMP) and related experiments. Through Ji's sum rule [4], one can, in fact, relate the components of the Energy Momentum Tensor (EMT) known as the gravitomagnetic form factors, $A_{q, g}$ and $B_{q, g}$, to the quark and gluon total angular momenta, $J_{q, g}$. The pivotal observation made in [4] is that $A_{q, g}$ and $B_{q, g}$ correspond to the $n=2$ Mellin moment of the GPDs which, in turn, define the matrix elements for DVCS. These important developments rendered total angular momentum a measurable quantity. Although the decomposition of $J_{g}$ into its spin and orbital components has proven to be hard to define gauge invariantly, the orbital angular momentum of quarks through, $J_{q}=L_{q}+S_{q}$, is well defined. Even so, the direct observability of $L_{q}$ remains a challenging question: the framework defined so far does not tell us how to access the dynamics of quark orbital motion since $L_{q}$ is only obtained through the difference of the total angular momentum and spin components.

Two alternative descriptions of OAM were derived more recently $[5,6,7,8,9]$. A dynamical picture of quark orbital motion was given in terms of a Generalized Transverse Momentum Distribution (GTMD), i.e. an unintegrated over transverse momentum GPD, in Ref.[6], while in Refs. [7, 8, 9] it was observed that OAM is associated to a twist three GPD. Notwithstanding these developments, two main problems remained to be solved: 1) relating the two distinct structures, one appearing at twist two and one at twist three, both describing of OAM within the same gauge invariant framework; 2) singling out an experimental measurement to access directly OAM, possibly through the newly defined structures. In this contribution we provide a link between the $k_{T}$ moment of the GTMD and the twist three GPD describing OAM. This enables us to single out, as a key measurement of OAM, the azimuthal angle modulations which are sensitive to twist three GPDs in DVCS off a longitudinally polarized target. Our observation is at variance with the expectation that transverse polarization, or proton spin flip processes are necessary to obtain information on quark OAM.

\section{A framework for measuring quark orbital angular momentum}

In what follows we present and discuss a critical link between two different definitions of OAM in the proton. The two definitions involve a GTMD called $F_{14}$ in the classification scheme proposed in [12], and a twist three GPD, $\tilde{E}_{2 T}$.

Quark OAM. $L_{q}$, enters the following gauge invariant decomposition of the proton's angular momentum [4],

$$
\begin{aligned}
& J_{q}+J_{g}=\frac{1}{2} \\
& J_{q}=S_{q}+L_{q}
\end{aligned}
$$


where $S_{q}$, is the quark spin summed over flavor, given by the integral of the helicity distribution, $g_{1}(x) ; J_{q(g)}$ is the quark (gluon) angular momentum given by the second moment of the unpolarized Generalized Parton Distribution (GPD) $H$ plus the spin flip GPD, $E$. Both $S_{q}$ and $J_{q}$ have been extracted from experiments (see Refs.[1,2] and [10], respectively). The extraction of $J_{q}$ has still a very large uncertainty and it is model dependent due to present limited kinematical coverage of experiments. $J_{q}$ has been calculated in lattice QCD: results on all quantities, $J_{q}, S_{q}$ and $L_{q}$, obtained by subtraction, are presented in Ref.[11].

$L_{q}$ has more recently been identified with precise operators and structure functions. On one side one has [6],

$$
L_{q}=\int d x \int d^{2} \mathbf{k}_{T} \int d^{2} \mathbf{b}\left(\mathbf{b} \times \overline{\mathbf{k}}_{T}\right)_{3} \mathscr{W}\left(x, \overline{\mathbf{k}}_{T}, \mathbf{b}\right),
$$

where $\mathscr{W}$ is a Wigner distribution given by the Fourier transform of the quark-quark off-forward unintegrated correlation function as,

$$
\mathscr{W}\left(x, \overline{\mathbf{k}}_{T}, \mathbf{b}\right)=\int \frac{d^{2} \Delta_{T}}{(2 \pi)^{2}} e^{i \mathbf{b} \cdot \Delta_{T}}\left[W_{++}^{\gamma^{+}}-W_{--}^{\gamma^{+}}\right]
$$

We have taken the same proton helicities in the initial and final states, $\Lambda=\Lambda^{\prime}$, of the correlator parametrized as in [12],

$$
\begin{aligned}
W_{\Lambda \Lambda}^{\gamma^{+}} & =\int \frac{d z^{-} d^{2} \mathbf{z}_{T}}{(2 \pi)^{3}} e^{i x P^{+} z^{-}-i \overline{\mathbf{k}}_{T} \cdot \mathbf{z}_{T}}\left\langle p^{\prime}, \Lambda\left|\bar{\psi}(-z / 2) \gamma^{+} \mathscr{U}(-z / 2, z / 2) \psi(z / 2)\right| p, \Lambda\right\rangle \\
& =\frac{1}{2 M} \bar{U}\left(p^{\prime}, \Lambda\right)\left(F_{11}+\frac{i \sigma^{i j} \bar{k}_{i} \Delta_{j}}{M^{2}} F_{14}\right) U(p, \Lambda)
\end{aligned}
$$

In Eqs.(2.3,2.4,2.5), $p=P+\Delta / 2, p^{\prime}=P-\Delta / 2, P=\left(p+p^{\prime}\right) / 2, k=\bar{k}+\Delta / 2, k^{\prime}=\bar{k}-\Delta / 2$, $\bar{k}=\left(k+k^{\prime}\right) / 2$, and the skewness parameter, $\xi=\Delta^{+} / P^{+}=0$, hence $t=\Delta^{2}\left(t \equiv-\Delta_{T}^{2}\right.$ for $\left.\xi=0\right)$; $\mathrm{U}(-\mathrm{z} / 2, \mathrm{z} / 2)$ is the gauge link. $F_{11}$ and $F_{14}$ are GTMDs describing an unpolarized quark inside an unpolarized proton, and an unpolarized quark inside a longitudinally polarized proton, respectively [12]. They depend on: $\left(x, \xi, \bar{k}_{T}^{2}, \bar{k}_{T} \cdot \Delta_{T}, t\right)$ while the GPDs depend on $(x, \xi, t) .{ }^{1}$ One can then relate $L_{q}$ to the $k_{T}$ moment of $F_{14}$ in the $\xi=0$ limit, through 2D Fourier transformation $[6,12,13]$,

$$
L_{q}=F_{14}^{(1)} \equiv \int_{0}^{1} d x \int d^{2} \overline{\mathbf{k}}_{T} \frac{\bar{k}_{T}^{2}}{M^{2}} F_{14}\left(x, 0, \bar{k}_{T}^{2}, \bar{k}_{T} \cdot \Delta_{T}, \Delta_{T}^{2}\right) .
$$

Another way of describing quark OAM was first introduced by Polyakov and collaborators in Ref.[7, 8], and subsequently rendered in a gauge field theory context in Ref.[9]. Using OPE it was shown that OAM can be described through a twist-three GPD, $G_{2}$, appearing in the parametrization of the twist three off-forward quark-quark correlation function (integrated over $k_{T}$ ),

$$
L_{q}=-\int_{0}^{1} d x x G_{2}=\frac{1}{2} \int_{0}^{1} d x x(H+E)-\frac{1}{2} \int_{0}^{1} d x \tilde{H} .
$$

\footnotetext{
${ }^{1}$ We follow the notation of Ref.[12], where $F_{1 n}(n=1,4)$, indicate twist-two GTMDs, and $F_{2 n}(n=1,8)$ twist-three GTMDs, respectively.
} 
In a nutshell, Eq.(2.7) was obtained by extending the derivation of the Efremov-Leader-Teryaev (ELT) sum rule [14] for the polarized structure functions to off-forward kinematics. By implementing the newer, uniform notation introduced in Ref.[12]) it can be shown that the twist three GPD $G_{2}$ corresponds to $\tilde{E}_{2 T}$ appearing in the twist three quark-quark off-forward correlation function (details on the two representations are given in [15]),

$$
\begin{aligned}
W_{\Lambda^{\prime} \Lambda}^{\gamma^{i}} & =\frac{M}{P^{+}} \frac{1}{2 P^{+}} \bar{U}\left(p^{\prime}, \Lambda^{\prime}\right)\left[i \sigma^{+i} H_{2 T}(x, \xi, t)+\frac{\gamma^{+} \Delta^{i}-\Delta^{+} \gamma^{i}}{2 M} E_{2 T}(x, \xi, t)\right. \\
& \left.+\frac{P^{+} \Delta^{i}-\Delta^{+} P^{i}}{M^{2}} \widetilde{H}_{2 T}(x, \xi, t)+\frac{\gamma^{+} P^{i}-P^{+} \gamma^{i}}{M} \widetilde{E}_{2 T}(x, \xi, t)\right] U(p, \Lambda) .
\end{aligned}
$$

It should be underlined that this derivation does not make use of GTMDs to describe OAM. We are therefore faced with an interesting situation where, in the context of Ji's decomposition of angular momentum, OAM can be described simultaneously by two different structure functions, a GTMD on one side, and a twist-three GPD on the other.

We report a preliminary result that a relation can be found between the two descriptions in terms of $F_{14}$, and $G_{2}$, respectively, that in turn, reflects both the underlying spin correlation and the gauge link structures of partonic OAM. The relation reads,

$$
\frac{d}{d x} \int d^{2} \bar{k}_{T} \frac{\bar{k}_{T}^{2}}{M^{2}} F_{14}=-G_{2} \Rightarrow \int_{0}^{1} d x \int d^{2} \bar{k}_{T} \frac{\bar{k}_{T}^{2}}{M^{2}} F_{14}=-\int_{0}^{1} d x x G_{2} \equiv L_{q} .
$$

Eq.(2.9) is a Lorentz Invariance Relation (LIR) [16], derived by observing that $F_{14}$, and $G_{2}$ (or similarly, $\tilde{E}_{2 T}$ ) admit a common substructure in terms of covariant amplitudes [12]. The procedure used to derive Eq.(2.9) is obtained following the approach originally used in Refs.[17] to derive Wandzura Wilczek (WW) type relations [18] for the twist-three distributions $g_{T}$ and $h_{L}$, and extending them to off-forward kinematics. The basis of our derivation is therefore also similar to previous work in that we use directly the nonlocal quark-quark and quark-gluon-quark correlators. This gives a more transparent interpretation than from using the standard methods of OPE, by emphasizing the role of partonic transverse momentum and off-shellness, whereby OAM is defined starting from nonlocal, $\bar{k}_{T}$ unintegrated, off-forward matrix elements.

The physical interpretation underlying this relation stems from the distinction between geometric/canonical twist and dynamical twist. Canonical twist, $\tau$, is defined formally as the canonical dimension minus the Lorentz spin of the local operators that enter the expansion of the various observables/currents in inverse powers of $Q^{2}$ (OPE). Dynamical twist, $t$, is defined by projecting out the quark field's good, $\gamma^{-} \gamma^{+} \psi$, and bad, $\gamma^{+} \gamma^{-} \psi$, components, respectively. The order of dynamical and canonical twist does not match beyond order two: contributions with the same power in $M / Q$, or same dynamical twist, can be written in terms of matrix elements of operators with different canonical twist, $\tau$. The WW relations between matrix elements of operators of different dynamical and same canonical twist encode this mismatch. Eq.(2.9) represents an extension of this physics to the off-forward case.

\section{OAM Observables}

The connection displayed in Eq.(2.9) uniquely enables us to perform an independent test of the quark OAM, $L_{q}$, spin, $S_{q}$, and total angular momentum, $J_{q}$, through separate observables. By 
providing a link between experiment and $k_{T}$ moments of specific GTMDs, it also allows us to make use of the newly developed method in lattice QCD for the calculation of TMDs [19, 20] to validate the angular momentum sum rules.

The observable to measure OAM through $G_{2}$ is the azimuthal asymmetry for DVCS of an unpolarized electron beam on a longitudinally polarized proton [21],

$$
A_{U L}=\frac{N_{s_{z}=+}-N_{s_{z}=-}}{N_{s_{z}=+}+N_{s_{z}=-}}
$$

where $N_{s_{z}= \pm}$ is a measure of the number of scatterings on a proton with longitudinal spin, $s_{z}=$ $\pm 1 / 2$. The dependence of $A_{U L}$ on the the angle $\phi$, or the azimuthal angle between the lepton plane and the plane of the virtual and real photons can be written keeping terms up to twist three as,

$$
A_{U L}=\frac{a \sin \phi+b \sin 2 \phi}{c_{0}+c_{1} \cos \phi+c_{2} \cos 2 \phi}
$$

where the coefficients for the total unpolarized cross section in the denominator, $c_{0}, c_{1}$, and $c_{2}$ are given by combinations of the Bethe-Heitler (BH), DVCS, and BH-DVCS interference terms [22]. The coefficients in the numerator, also displayed in [22] contain the GPDs of interest in our study through the associated Compton form Factors, at twist two, $\widetilde{\mathscr{H}} \rightarrow \tilde{H}$ and at twist three $\widetilde{\mathscr{H}}^{\text {eff }} \rightarrow G_{2}$,

$$
a \approx s_{1, L P}^{I} \propto F_{1}(t) \mathfrak{I} m \widetilde{\mathscr{H}}
$$

and

$$
b \approx s_{2, L P}^{I} \propto F_{1}(t) \mathfrak{I} m \widetilde{\mathscr{H}}^{e f f}
$$

where $F_{1}(t)$ is the Dirac form factor. The asymmetry is shown in Fig.1 plotted vs. the momentum transfer squared $-t$, compared to HERMES data [23]. Both the twist two $(\sin \phi)$ and twist three $(\sin 2 \phi)$ modulations are shown. The blue bands represent the predictions from the GPD parametrization of $[24,25]$ including the error from the model's parameters variations. The GPD $G_{2}$ was calculated within the WW approximation. As we can see from the figure the $\sin 2 \phi$ modulation, dominated by the $\tilde{\mathscr{H}}$ Compton form factor, is sizable. An extraction of $G_{2}$ will be possible at Jefferson Lab@12 GeV making use of a more extensive kinematical coverage in $x_{B j}$ and $Q^{2}$ than the present kinematical range.

\section{Conclusions and Future Developments}

In conclusion, we provided a relation that is key for addressing the question of a direct measurement of OAM in the proton. The underpinnings of a field theoretic rendition of OAM in QCD are best captured by our relation which brings to the forefront the intricacies of connecting a twist two GTMD moment and a twist three GPD. By implementing directly non local, $k_{T}$-unintegrated quark-quark correlation functions our approach opens up an avenue to explore the role of partonic transverse momentum and off-shellness in OAM, while providing a formalism which connects to lattice QCD calculations on one side and to experiment on the other.

Our relation was shown in the case of a straight gauge link only, or for Ji's decomposition of angular momentum. Future developments include the extension of our study to the Jaffe Manohar 


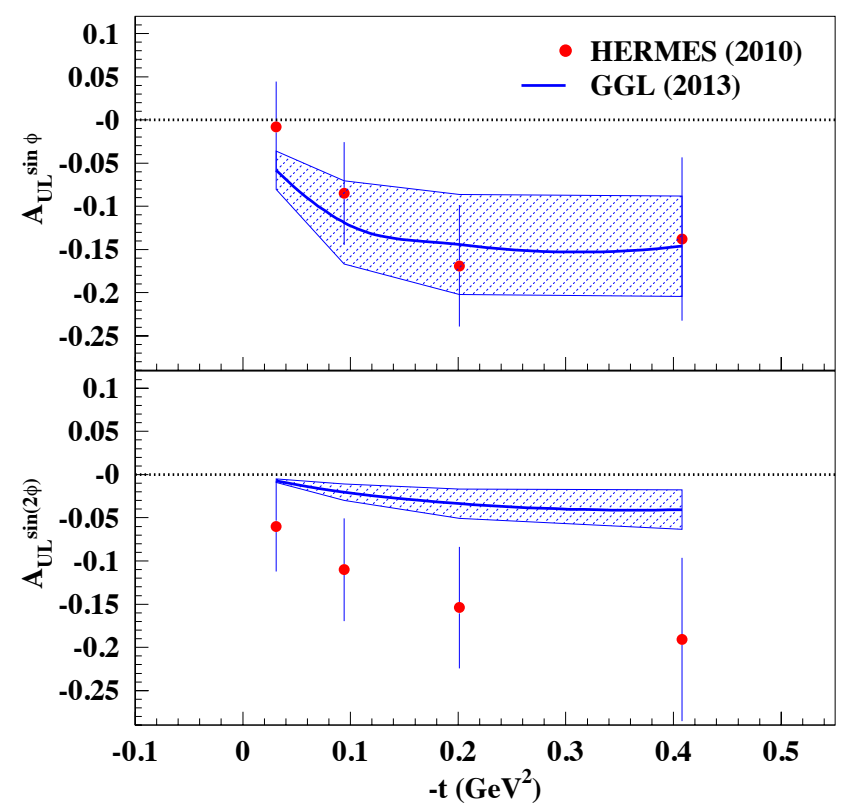

Figure 1: Adapated from Ref.[21]. The asymmetry $A_{U L}$ twist two $(\sin \phi)$ and twist three $(\sin 2 \phi)$ modulations plotted vs. the momentum transfer squared $-t$, compared to HERMES data [23] at the Bjorken $x$ and scale $Q^{2}$ of the data. The blue bands represent the predictions from the GPD model of [24, 25] denoted by GGL in the legend, including the error from the model's parameters variations calculated in WW approximation.

[26] decomposition which, as shown in Ref.[27] involves a final state interaction, and is related to the Ji decomposition as follows,

$$
L_{q}^{\mathrm{JM}}=L_{q}^{\mathrm{Ji}}+\langle\tau\rangle
$$

where

$$
\begin{aligned}
\left\langle\tau_{z}\right\rangle & ==\int d z^{-} d^{2} \mathbf{z}_{T}\left\langle p, \Lambda\left|\bar{\psi}(\mathbf{z}) \gamma^{+} \int_{z^{-}}^{\infty} d z^{\prime-} \tau_{z}\left(z^{\prime-}, \mathbf{z}_{T}\right) \psi(\mathbf{z})\right| p, \Lambda\right\rangle \\
\tau_{z} & =-g\left(x G^{+2}-y G^{+1}\right)
\end{aligned}
$$

is an off-forward extension of a Qiu-Sterman term [28]. This appears to have the physical meaning of a torque (a final state interaction) exerted on the outgoing quark by the color-magnetic field produced by the spectators [27].

The results presented here will appear discussed in detail in a forthcoming publication [15].

\section{References}

[1] J. Ashman et al. [European Muon Collaboration], Phys. Lett. B 206, 364 (1988). doi:10.1016/0370-2693(88)91523-7

[2] J. Ashman et al. [European Muon Collaboration], Nucl. Phys. B 328, 1 (1989). doi:10.1016/0550-3213(89)90089-8 
[3] A. V. Radyushkin, Phys. Rev. D 56, 5524 (1997)

[4] X. D. Ji, Phys. Rev. Lett. 78, 610 (1997)

[5] M. Burkardt and H. BC, Phys. Rev. D 79, 071501 (2009); Few Body Syst. 52, 389 (2012).

[6] C. Lorce and B. Pasquini, Phys. Rev. D 84, 014015 (2011)

[7] M. Penttinen, M. V. Polyakov, A. G. Shuvaev and M. Strikman, Phys. Lett. B 491, 96 (2000)

[8] D. V. Kiptily and M. V. Polyakov, Eur. Phys. J. C 37, 105 (2004)

[9] Y. Hatta and S. Yoshida, JHEP 1210, 080 (2012)

[10] M. Mazouz et al. [Jefferson Lab Hall A Collaboration], Phys. Rev. Lett. 99, 242501 (2007) [arXiv:0709.0450 [nucl-ex]].

[11] J. D. Bratt et al. [LHPC Collaboration], Phys. Rev. D 82, 094502 (2010) doi:10.1103/PhysRevD.82.094502 [arXiv:1001.3620 [hep-lat]].

[12] S. Meissner, A. Metz and M. Schlegel, JHEP 0908, 056 (2009)

[13] Y. Hatta, Phys. Lett. B 708, 186 (2012) doi:10.1016/j.physletb.2012.01.024 [arXiv:1111.3547 [hep-ph]].

[14] A. V. Efremov, O. V. Teryaev and E. Leader, Phys. Rev. D 55, 4307 (1997) doi:10.1103/PhysRevD.55.4307 [hep-ph/9607217].

[15] A. Rajan, A. Courtoy, M. Engelhardt and S. Liuti, in preparation.

[16] A. Bacchetta, M. Diehl, K. Goeke, A. Metz, P. J. Mulders and M. Schlegel, JHEP 0702, 093 (2007)

[17] P. J. Mulders and R. D. Tangerman, Nucl. Phys. B 461, 197 (1996) [Nucl. Phys. B 484, 538 (1997)]

[18] S. Wandzura and F. Wilczek, Phys. Lett. B 72, 195 (1977).

[19] B. U. Musch, P. Hägler, M. Engelhardt, J. W. Negele and A. Schäfer, Phys. Rev. D 85, 094510 (2012); M. Engelhardt et al., PoS LATTICE 2014, 167 (2014).

[20] M. Engelhardt et al., PoS LATTICE 2014, 167 (2014).

[21] A. Courtoy, G. R. Goldstein, J. O. G. Hernandez, S. Liuti and A. Rajan, Phys. Lett. B 731, 141 (2014); arXiv:1412.0647 [hep-ph].

[22] A. V. Belitsky, D. Mueller and A. Kirchner, Nucl. Phys. B 629, 323 (2002) [hep-ph/0112108].

[23] A. Airapetian et al. [HERMES Collaboration], JHEP 1006, 019 (2010)

[24] G. R. Goldstein, J. O. Hernandez and S. Liuti, Phys. Rev. D 84, 034007 (2011)

[25] J. O. Gonzalez-Hernandez, S. Liuti, G. R. Goldstein and K. Kathuria, Phys. Rev. C 88, no. 6, 065206 (2013) doi:10.1103/PhysRevC.88.065206 [arXiv:1206.1876 [hep-ph]].

[26] R. L. Jaffe and A. Manohar, Nucl. Phys. B 337, 509 (1990).

[27] M. Burkardt, Phys. Rev. D 88, no. 1, 014014 (2013)

[28] J. Qiu and G. F. Sterman, Phys. Rev. Lett. 67, 2264 (1991). 